\title{
Transferring endophyte technology to North American farmers
}

\author{
J.G. ANDRAE ${ }^{1}$ and C.A. ROBERTS 2 \\ ${ }^{I}$ Clemson University, Clemson SC USA \\ ${ }^{2}$ University of Missouri, Columbia MO USA \\ jandrae@clemson.edu
}

\begin{abstract}
Bacon and others discovered the tall fescue endophyte in 1977. Since that time, North American researchers have developed several endophyte-based technologies to reduce fescue toxicosis. The most recent has been the release of MaxQ, a novel endophyte that enhances persistence but does not cause fescue toxicosis. Despite its advantages, MaxQ has been adopted slowly across the fescue belt of the US. This paper presents possible social and managerial reasons for this slow adoption. It closes by offering some possible solutions.
\end{abstract}

Keywords: MaxQ, novel endophyte, tall fescue toxicosis, pasture renovation, educational efforts

\section{Introduction}

The presence of the wild-type endophyte in tall fescue and its impacts on cattle production has been known for many years throughout the tall fescue belt of the U.S., but adoption of new technology to alleviate tall fescue toxicosis has been slow. The slow adoption rate is surprising, particularly since the impacts of tall fescue toxicosis are visually apparent and economically important. This paper identifies some of the reasons for the slow technology adoption and offers suggestions for improving adoption rates. The following observations and suggestions are based mainly upon personal interactions with farmers and grazing operations throughout the tall fescue belt of the United States in educational meetings, farm visits and workshops. Observations are also based on feedback from many extension specialists in the region over the past several years, which this paper attempts to incorporate.

Why are there Problems Adopting Endophyte Technology? It is surprising that only a small proportion of North American tall fescue hectares have been converted to non-toxic tall fescue over the past 5 years. Most farms in the tall fescue belt contain monocultures of toxic fescue, which provides the sole source of hay and pasture for animals year-round. Producers in this region have been "trained" over the past 20 years to recognise the grotesque clinical signs of fescue toxicosis, such as lost tail switches and fescue foot. However, they have not been trained to recognise the less visible symptoms of tall fescue toxicosis, such as low rate of gain.

It is possible that the educational success of the extension professional in diagnosing the obvious symptoms of tall fescue toxicosis may be counterproductive to the alleviation of this problem over the long term. Producers seem now to be searching for the most severe symptoms of tall fescue toxicosis but "can't see the forest because of all the trees". Since the region is completely covered with toxic tall fescue, every producer's cattle herd looks similar to his neighbour's cows. Calf weight gains are rarely monitored on the farm, so there is no way to calculate actual rate of gain. There is no "control" group grazing nontoxic fescue across the fence, so there is no opportunity to compare affected animals to healthy animals. As a result, fescue toxicosis is perceived as a malady that affects neighbours and farmers in nearby counties, while "I don't have a problem with it on my farm."

There are other reasons producer adoption of endophyte technology may be slow. One reason is herd size. The mean herd size for most states in the fescue belt ranges from 18-38 head per farm (Table 1). The majority of farms in this region contain fewer than 50 head of cattle (USDA 2002). Compare these herds to those in the Western U.S. or Canada, where many ranches are medium to large (500 head of cattle or greater). Herds of the medium to large size in the fescue belt make up only $1 \%$ of total beef cattle operations.

Table 1 Cattle inventory, mean herd size and proportion of farms containing less than 50 head and greater than 500 head in selected states of the tall fescue belt. Data adapted from USDA National Agricultural Statistics Service, 2002.

\begin{tabular}{|c|c|c|c|c|}
\hline State & $\begin{array}{l}\text { Cattle and calf } \\
\text { inventory (x1000) }\end{array}$ & $\begin{array}{l}\text { Mean herd size per } \\
\text { farm }^{\star}\end{array}$ & $\begin{array}{c}\text { Farms with }<50 \\
\text { hd, } \%\end{array}$ & $\begin{array}{c}\text { Farms with }>500 \\
\text { hd, } \%\end{array}$ \\
\hline Alabama & 1,438 & 29 & 73 & $1 v$ \\
\hline Arkansas & 1,842 & 38 & 66 & $<1$ \\
\hline Georgia & 1,272 & 26 & 75 & 1 \\
\hline Kentucky & 2,395 & 26 & 73 & $<1$ \\
\hline Mississippi & 1,072 & 25 & 75 & $<1$ \\
\hline Missouri & 4,460 & 34 & 62 & 1 \\
\hline North Carolina & 848 & 18 & 83 & $<1$ \\
\hline South Carolina & 432 & 19 & 79 & $<1$ \\
\hline Tennessee & 2,234 & 20 & 77 & $<1$ \\
\hline Virginia & 1,623 & 31 & 71 & 1 \\
\hline
\end{tabular}

* data in column derived from 1995 Cattle Industry Reference Guide, C-F Industries Englewood CO, USA. 
Figure 1 Flow chart showing approach used in Missouri to deal with fescue toxicosis. This approach is presented in 2-day toxicosis workshops.

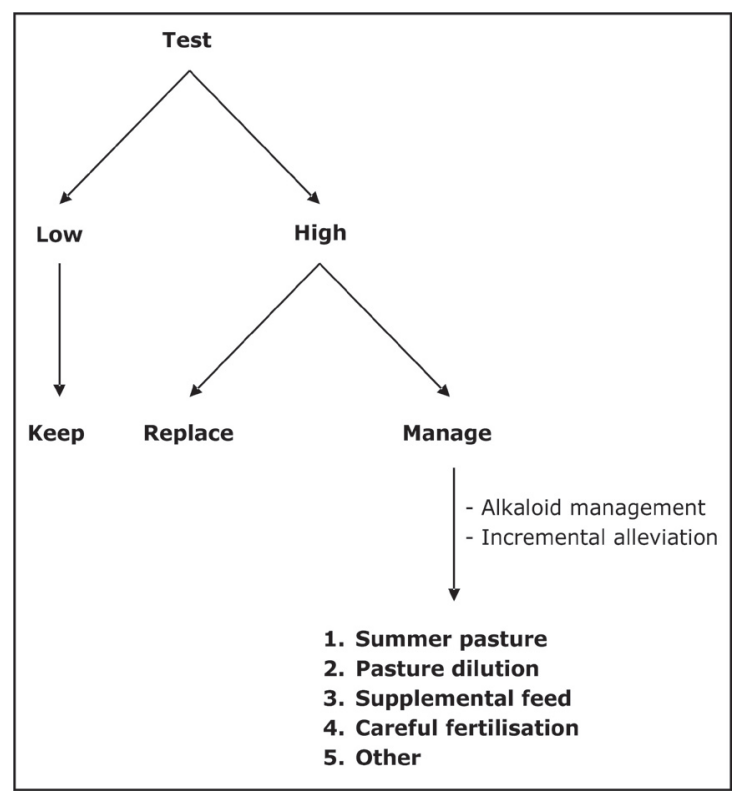

Some colleagues have suggested that convincing small farmers to replace toxic tall fescue should be easier than convincing larger operations. After all, there are fewer hectares to convert in small operations, and the planning process should be simplified with smaller herds. In reality, the opposite is true. Most small operations are managed or owned by part-time farmers who have limited funds budgeted to renovate pastures and limited equipment with which to spray and plant. Many farms also have limited cross fencing and water, which makes it difficult to rotate animals to alternate pastures during renovation.

Another reason for slow adoption of endophyte technology relates to rental agreements. Many farmers in the fescue belt of the U.S. rent pastureland on a year-to-year basis, with no guarantee of future contracts. The lack of long-term rental contracts discourages long-term planning, and makes even the most common routine agronomic practices such as liming appear financially risky. To complicate matters, much of the pastureland in this region is steep and unsuited for tillage.

Finally, a reason for slow adoption relates to the social environment in North America. Many U.S. cattlemen consider themselves cowboys, not grasslanders. This difference has been acknowledged in past conferences, but it needs to be reiterated; it cannot be over-emphasised. The American cowboy's primary management interest emphasises the "animal" component of animal production, and often completely ignores grazing management. The majority of cattlemen in North America find it perfectly logical to purchase a $\$ 4,000$ bull, yet ludicrous to eradicate a "good tall fescue pasture" (even if toxic), only to reestablish another stand of tall fescue.

\section{Educational Outreach Efforts}

Regarding the toxicosis management message itself, it seems that extension specialists are offering science-based solutions and saying the right things. Most forage and livestock specialists are in agreement regarding individual management practices, and they are telling people about the novel endophytes. However, there continue to be exceptions.
For example, in July of 2006, an article circulated through a popular mid-America publication contained the following management recommendations: if the pasture is endophyteinfected Kentucky 31, the producer should renovate the pasture or replace it with another grass (including MaxQ), graze pastures low or clip, feed minerals, feed seaweed, avoid calving and breeding in the summer, and conduct an endophyte test. The article placed high value on feeding certain additives with a questionable effect on livestock performance, yet it omitted one of the primary recommendations - to provide alternative species for summer pasture. The fact that the article was attributed to a university specialist muddles the extension message.

Regarding message delivery, American extension efforts could benefit from a different and more comprehensive vehicle. Most messages are delivered via phone interviews, articles in the popular press, and after supper talks. Such vehicles provide brief and simplistic dialog when a much more in-depth approach is needed. In short, when discussing fescue toxocosis and management, American educators often "bring a knife to a gunfight." The after supper talks cannot facilitate persuasive or detailed discussions on fescue toxicosis. Such detailed discussions are needed, as fescue toxicosis is a complex syndrome that is addressed by over 100 confusing and ineffective home remedies (Roberts \& Andrae 2005). A better delivery system would allow the message to describe the cause of toxicosis, the ubiquitous nature of problem, and the full range of science-based recommendations.

\section{Focusing on the Positive: Opportunities for Increased Adoption}

The limited size of many farms in the tall fescue belt was previously listed as a disadvantage; however, the $1 \%$ of farms containing herds greater than 500 head of beef cattle represents approximately $15 \%$ of the total cattle in the region (USDA 2002). Translated simply, the largest gains in endophyte adoption can be made by intensely focusing outreach and marketing efforts on large, business-oriented farmers. These farmers are likely to see the huge "bang for your buck" potential. And they have the resources and profit-based motivation to renovate their pastures. Their adoption will hopefully encourage smaller cattleman to renovate pastures in future years.

Farmers appear to have few, if any, doubts about animal performance improvements when pastures are converted to MaxQ tall fescue. This could be due to successful endophytefree tall fescue demonstrations and limited producer experiences during the 1980s. Unfortunately, the same farmers recall the decreased persistence of endophyte-free tall fescue, which surprised researchers and industry. During the 1980s, many producers wholeheartedly adopted endophyte-free tall fescue only to have complete stand failures within a few years. This experience has almost certainly left them wary of persistence data comparing MaxQ and toxic tall fescue. To alleviate producer (as well as extension) concerns regarding MaxQ persistence, over 150 demonstration plots were established throughout the tall fescue belt by Pennington seed and extension personnel. These demonstrations, coupled with word-of-mouth testimonials from early adopting producers, have demonstrated to farmers under local conditions that MaxQ tall fescue does indeed possess similar stand persistence as toxic tall fescue. The favorable agronomic performance of these demonstration plots at several sites under severe drought has also helped to quiet producer concerns regarding decreased persistence of nontoxic endophyteinfected tall fescue. It appears that animal performance and plant persistence issues are being resolved at the producer level. 
Even though limited concern remains, the majority of future outreach efforts should "move past" traditional demonstrations that address persistence and animal performance and focus on producer concerns currently inhibiting adoption.

Some of the variables influencing a producer's decision to plant a novel endophyte are based on understandable factors. Six of these were published in an electronic guide (Roberts \& Andrae 2004) and include level of toxic endophyte, land ownership, landscape, livestock class, grazing management, and seed production. To illustrate these factors, the most unlikely person who would replant would be a cow-calf operator who is renting land on steep hillsides of tall fescue infected with $15 \%$ endophyte, yet with no land for cattle to graze during establishment of MaxQ.

Other specific producer concerns were ranked by extension specialists and reported by Roberts \& Andrae (2005) at the Arkansas symposium. The top predicted producer "drawbacks" (in order of importance) were (1) seed cost (2) process of killing old and planting new (3) not good investment on rented land and (4) pasture reverting back to toxic fescue. At least two of these concerns have since been addressed with research trials. Results from these trials need to be effectively delivered to cattlemen in the tall fescue belt.

The potential for pastures to "revert" to toxic tall fescue is a concern often voiced by producers. Reversion was addressed by Barker et al. (2005). Experimental seedings were contaminated with known proportions of toxic tall fescue and monitored over time. Mixtures containing endophyte-free seed showed higher than expected proportions of toxic tall fescue contamination whereas novel endophyte-infected tall fescue maintained expected levels of contamination. This suggests that, at least in the upper reaches of the tall fescue belt, novel endophytes can compete well with toxic tall fescue seedlings and resist further encroachment of these seedlings. Delivering this research information to producers in a timely and effective manner should reinforce the favorable agronomic characteristics of MaxQ tall fescue and present the relatively low risk of recontamination in properly managed pastures.

Replacing toxic tall fescue stands with non-toxic MaxQ was the second factor predicted to limit producer adoption. Alternatives to the spray-smother-spray procedure have since been investigated and modified to allow near complete eradication of toxic tall fescue with spring mowing and double applications of glyphosate in the fall (Andrae et al. 2005). This protocol has also been effectively demonstrated on farms in the extreme Southeast portion of the tall fescue belt; however, it remains untested in more westerly and northern areas where herbicide efficacy and seed bank dynamics may differ. In a broader sense, several different methods used to replace toxic tall fescue with MaxQ should be evaluated alongside other options for managing existing toxic tall fescue using a systems-oriented approach. Individual farm circumstances differ and these differences may dramatically alter the appropriate proportion of pastures and/or methods used to renovate these pastures. A more comprehensive, integrated model is necessary for effective delivery of tall fescue toxicosis management.

Tall fescue Toxicosis and Management Workshop: a New Delivery Model for Endophyte Education

One activity that could increase adoption of endophyte technology is the toxicosis workshop. The workshop was proposed in 2004 (Roberts \& Andrae 2004) and was subsequently organised by University of Missouri (Roberts \& Crawford) and University of Georgia (Andrae).
The toxicosis workshop, officially called "Fescue Toxicosis and Management Workshop," differs from routine extension efforts. It was patterned after the University of Missouri Grazing Schools, originally developed by Jim Gerrish and Ron Morrow. The workshop uses 2 days to focus attention on the toxicosis problem and offer solutions for improving management and increasing profitability of forage-livestock operations in the upper South and lower Midwestern US. The intense, 2-day format is more effective than a series of disconnected supper talks. This workshop is "pay per view," with an entry fee of \$150 USD.

\section{Success of the workshop}

The success of this workshop depends on several aspects, the first being order of information. The workshop begins by confronting the great denial - "I don't have an endophyte problem because 1) I have legumes and 2) my cattle do not lose tails and hooves." The initial session shows that fescue toxicosis occurs on at least $80 \%$ of the farms in which Kentucky 31 is grazed. The effectiveness of this initial session is most important, as it sets the stage for solutions to follow.

The success of the workshop also depends on reliable data. Data are important in all agricultural workshops. But they are doubly important when discussing fescue toxicosis, a syndrome often attributed to sensational phenomena and addressed in coffee shop recommendations - breeding and feeding. The data used in this workshop are similar to those published in the electronic guide (Roberts \& Andrae 2004).

The success of the workshop also depends on demonstrations. For each major point in the workshop, a demonstration is offered. The most important demonstration is the brief sideby-side comparison of cattle that have grazed toxic or nontoxic pastures. This demonstration shows livestock producers what researchers see daily-rough hair coat, unthrifty appearance, excessive nervousness and urination, and usually, low body condition score. The visual and typically striking comparison of herd mates who have grazed toxic or non-toxic tall fescue helps to show farmers what they often cannot admit; fescue toxicosis exists on their farm. This recognition is a critical initial step in the education process.

Workshop success also depends on economic analysis. This analysis involves partial budgets, because complete analysis based on the total farm often only confuses our producers. The economic analysis is presented after each science-based talk in the classroom and demonstration in the field. It is the final exercise in a three-step process we call "data-demo-dollars."

Lastly, the success of this workshop depends on presentation of a grazing system. Over the years, many of us have responded to questions on fescue toxicosis with a component-based thinking. We have suggested one or two recommendations, such as interseeding legumes or supplementing the diet. The systems approach allows producers to understand benefits of seasonlong, additive practices. Therefore, the workshop promotes and concludes with an in-depth discussion of a complete pasture management system; this discussion is intended to be especially beneficial to those producers who choose to retain their Kentucky 31.

The two underlying concepts in this discussion are incremental alleviation and alkaloid management. The concept of incremental alleviation states that, should toxic tall fescue be managed instead of replaced, toxicosis can be alleviated by a series of practices that result in incremental improvement in animal performance. For example, interseeding of legumes might add between 0.1 and $0.14 \mathrm{~kg} /$ day to a steer ADG; it would only be, however, a 
single component in a system that incorporated several other practices. The concept of alkaloid management states that livestock and pastures must be managed to minimise ingestion of ergot alkaloids. Standard management is based on yield, as livestock are rotated among fields in response to forage on offer. Standard management does not address alkaloid concentration, and it often consists of responding to alkaloids that were previously ingested.

The grazing system we present uses a two-pasture operation that includes tall fescue and bermudagrass. The fescue-based fields provide spring and fall pasture, and the bermudagrass fields provide summer pasture. Bermudagrass fields also provide winter pasture, as they are overseeded with cereal grass in the early fall. The grazing policy requires livestock to be moved to bermudagrass as soon as possible, which in Missouri, occurs while tall fescue is in the boot stage. Although the early move leaves behind a considerable supply of non-grazed forage, this material can be harvested as hay. The early move emphasises the concept of alkaloid management and allows livestock to bypass the tall fescue pasture when it is most toxic, during reproductive development that occurs in late spring.

\section{Workshop take-home message}

The workshop offers a take-home message-fescue toxicosis must be addressed methodically. It offers an approach (Fig. 1) that begins with endophyte testing. If pastures are found to contain high levels of endophyte ( $>55 \%)$, the approach calls for producers to consider replanting to a nontoxic grass or adopting a management scheme for highly infected Kentucky 31. Of course, the approach assumes that all pastures, including those with low endophyte levels and novel endophyte, would be managed. But it calls for a particular management philosophy in the case of toxic tall fescue. That philosophy involves the two concepts mentioned above-incremental alleviation and alkaloid management.

Without this complete approach, producers do not typically test for endophyte and tend to follow only one or two suggestions to alleviate toxicosis, often incorporating some methods that are not recommended. But with the integrated workshop approach (Fig. 1), producers see what is involved in proper management of infected Kentucky 31. It is our hope that with proper comprehensive education, many producers might opt for replanting to a novel endophyte, thereby reducing input costs required for incremental management of toxic tall fescue.

\section{Results of the workshops}

The 2004 evaluations were more favourable than those we have for the Missouri Grazing Schools. The 2005 evaluations were favourable as well. We do not know if these successes will continue, but we are hoping to ensure such by making improvements in the school. These improvements include a specific curriculum with scripted PowerPoint presentations and a tracking process to document impact. We do not yet have in place this tracking process, so we do not know how many producers leave the workshop and plant MaxQ.

\section{Summary}

If endophyte technology is to be adopted in the fescue belt of the US, educational efforts must begin by persuading producers of the seriousness of fescue toxicosis, namely, lower rate of gain, calving percentage, milk production, and other aspects of herd health. Producers do not believe the problem is as serious as it really is. Future educational efforts should also strive to employ a comprehensive educational program, not a piecemeal of component-based messages. Additionally, producers should see a consistent message delivered from extension, seed companies, consultants, innovative farmers, and agency personnel. This program should specifically focus on managing toxins in tall fescue pastures.

\section{REFERENCES}

Andrae, J.G.; Hill, N.S.; Durham, R.G. 2005. Methods to replace toxic tall fescue with

MaxQ tall fescue. Are summer smother crops necessary? p.70 In: Vol. 14 of the American Forage and Grassland Council Proceedings. Bloomington, IL.

Barker, D.J.; Sulc, R.M.; Bultemeier, T.L.; McCormick, J.S.; Little, R.; Penrose, C.D.; Samples, D. 2005. Contrasting toxic-endophyte contamination between endophyte-free and nontoxic-endophyte tall fescue pastures. Crop Science 45: 616-625.

Roberts, C.A.; Andrae, J.G. 2004. Tall fescue toxicosis and management. Online. Crop Managm. doi. 10:1094/CM-20040427-01-MG.

USDA, 2002. National Agricultural Statistics Service 2002 Census. (Available online at http://www.nass.usda.gov/ QuickStats/Create_Federal_All.jsp; verified 12 Dec. 2006).

Roberts, C.A.; Andrae, J.G. 2005. Public education in tall fescue toxicosis. pp. 359-377. In: Neotyphodium in CoolSeason Grasses. Eds. Roberts, C.A.; West, C.P.; D.E. Sp iers, 